\title{
Free degrees of homeomorphisms on compact surfaces
}

\author{
JIANCHUN WU \\ XUEZHI ZHAO
}

\begin{abstract}
For a compact surface $M$, the free degree $\mathfrak{f r}(M)$ of homeomorphisms on $M$ is the minimum positive integer $n$ with property that for any self homeomorphism $\xi$ of $M$, at least one of the iterates $\xi, \xi^{2}, \ldots, \xi^{n}$ has a fixed point. This is to say $\mathfrak{f r}(M)$ is the maximum of least periods among all periodic points of self homeomorphisms on $M$. We prove that $\mathfrak{f r}\left(F_{g, b}\right) \leq 24 g-24$ for $g \geq 2$ and $\mathfrak{f r}\left(N_{g, b}\right) \leq 12 g-24$ for $g \geq 3$.
\end{abstract}

$55 \mathrm{M} 20 ; 37 \mathrm{E} 30$

\section{Background}

Let $M$ be a compact surface and $\xi$ be a self homeomorphism on $M$. The free degree $\mathfrak{f r}(\xi)$ of $\xi$ is the maximum positive integer $n$ such that $\xi^{1}, \xi^{2}, \ldots, \xi^{n-1}$ are all fixed point free. For a set $S$ which consists of self homeomorphisms on $M$, we denote the free degree of $S$ by $\mathfrak{f r}(S)=\max \{\mathfrak{f r}(\phi) \mid \phi \in S\}$. We denote the free degree of all homeomorphisms by $\mathfrak{f r}(M)$ and the free degree of all orientation preserving homeomorphisms by $\mathfrak{f r}^{+}(M)$.

We write $F_{g}$ for an orientable closed surface of genus $g$ and $N_{g}$ for a nonorientable closed surface of genus $g$ (ie a connected sum of $g$ projective planes), write respectively $F_{g, b}$ and $N_{g, b}$ for an orientable and nonorientable surface with $b$ boundary components.

J Nielsen [4] studied $\mathfrak{f r}^{+}\left(F_{g}\right)$ in the 1940s, showing that

$$
\mathfrak{f r}^{+}\left(F_{g}\right)= \begin{cases}2 \text { or } 3 & \text { if } g=2, \\ 2 g-2 & \text { if } g>2 .\end{cases}
$$

The exact value $\mathfrak{f r}^{+}\left(F_{2}\right)=2$ was determined by W Dicks and J Llibre [1] in 1996.

In the 1990s, S Wang [7] obtained results on all homeomorphisms and on nonorientable closed surfaces. One of his results is

$$
\mathfrak{f r}\left(F_{g}\right)= \begin{cases}4 & \text { if } g=2, \\ 2 g-2 & \text { if } g>2\end{cases}
$$


In this paper, we consider $\mathfrak{f r}(M)$ when $M$ is a connected compact surface with boundaries. Our main result is:

Theorem 1.1 For $F_{g, b}$ and $N_{g, b}$ orientable and nonorientable genus $g$ surfaces with $b$ boundary components, the free degrees satisfy:

$$
\begin{gathered}
\max _{b} \mathfrak{f} \mathfrak{r}\left(F_{g, b}\right) \begin{cases}=\infty & \text { if } g=0,1, \\
\leq 24 g-24 & \text { if } g \geq 2 .\end{cases} \\
\max _{b} \mathfrak{f r}\left(N_{g, b}\right) \begin{cases}=\infty & \text { if } g=1,2, \\
\leq 12 g-24 & \text { if } g \geq 3 .\end{cases}
\end{gathered}
$$

This means that for given $g$, the free degree $\mathfrak{f r}\left(F_{g, b}\right)$ and $\mathfrak{f r}\left(N_{g, b}\right)$ have an uniform upper bound which is independent of the number of boundary components.

\section{Nielsen fixed point theory}

In this section, we shall review some facts in Nielsen fixed point theory; see Jiang [2] for more details.

Given any self map $f: X \rightarrow X$, the fixed point set of $f$ is divided into a disjoint union of some subsets, each is said to be a fixed point class of $f$. A fixed point class is an isolated fixed point set, and hence has well-defined fixed point index. The sum of all indices is the Lefschetz number $L(f)$. The number of essential (nonzero indices) fixed point class is defined to be the Nielsen number $N(f)$. One of the key result in Nielsen fixed point theory is:

Proposition 2.1 (Jiang [2, page 19, 4.7 Theorem]) Let $X$ be a compact polyhedron. Then, any self-map in the homotopy class of $f: X \rightarrow X$ has at least $N(f)$ fixed points.

This result refines the Lefschetz fixed point theorem: $L(f) \neq 0$ implies the fixed point set of $f$ is nonempty.

Apply these basic properties of this two invariants. We have the following.

\section{Proposition 2.2}

$$
\mathfrak{f r}(f) \leq \min \left\{n \mid N\left(f^{n}\right)>0\right\} \leq \min \left\{n \mid L\left(f^{n}\right) \neq 0\right\} .
$$

This proposition is one of main tool in our present paper. 


\section{Standard forms of surface homeomorphisms}

According to Nielsen-Thurston classification theorem of surface homeomorphisms, any surface homeomorphism is isotopic to either a periodic, pseudo-Anosov or reducible one (see Thurston [5]). In this section, we recall the "standard" homeomorphisms introduced by B Jiang and J Guo [3]. Some adjustments are made for our purpose. The local behavior at periodic points is addressed.

Let $p$ be a positive integer, $k$ an integer, and $\lambda$ a real number with $\lambda>1$. We define

(1) $r_{(p, k, \lambda)}^{+}$: a self map on $\mathbb{C}$ given by $r_{(p, k, \lambda)}^{+}\left(\rho e^{\theta i}\right)=\rho e^{(\theta+2 k \pi / p) i}$;

(2) $r_{(p, k, \lambda)}^{-}$: a self map on $\mathbb{C}$ given by $r_{(p, k, \lambda)}^{-}\left(\rho e^{\theta i}\right)=\rho e^{-(\theta+2 k \pi / p) i}$;

(3) $\eta_{(p, k, \lambda)}$ : a self map on $\mathbb{C}$ which is the time-one map of the vector field $v$ defined by $v\left(\rho e^{\theta i}\right)=(2 \ln \lambda / p) \rho e^{(1-p) \theta i}$;

(4) $\eta_{(p, k, \lambda)}^{\prime}$ : a self map on $\mathbb{C}-\operatorname{int}(D)$ which is the time-one map of the vector field $v^{\prime}$ defined by $v^{\prime}\left(\rho e^{\theta i}\right)=(2 \ln \lambda / p)\left((\rho-1) e^{(1-p) \theta i}+e^{(\theta-\pi / 2) i} \sin (p \theta)\right)$, where $D$ is the unit disk in complex plane $\mathbb{C}$.

Lemma 3.1 [3, 2.1] Let $\psi$ be a pseudo-Anosov homeomorphism on a compact surface $F$, having stable foliation $\mathcal{F}^{s}$ and unstable foliation $\mathcal{F}^{u}$ with dilatation $\lambda$. Then there is a smooth atlas $\mathcal{U}$ of $F$, consisting of one chart for each interior singularity, one chart for each boundary component, and some other charts at regular point, such that

(1) if $u_{x}:\left(U_{x}, x\right) \rightarrow(\mathbb{C}, 0)$ is the chart for an interior singularity $x$, then the prongs of $\mathcal{F}^{s}$ are $\left\{u^{-1}\left(\rho e^{(m \pi / p) i}\right) \mid \rho \geq 0, m=1,3,5, \ldots, 2 p-1\right\}$, the prongs of $\mathcal{F}^{u}$ are $\left\{u^{-1}\left(\rho e^{(m \pi / p) i}\right) \mid \rho \geq 0, m=0,2,4, \ldots, 2 p-2\right\}$, and there is a commutative diagram

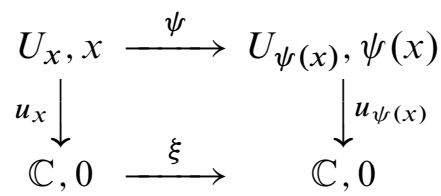

where $\xi=r_{(p, k, \lambda)}^{+} \circ \eta_{(p, k, \lambda)}$ or $\xi=r_{(p, k, \lambda)}^{-} \circ \eta_{(p, k, \lambda)}$ for some nonnegative integer $k$ (the singularity $x$ is said to be of type $(p, k)^{+}$or of type $\left.(p, k)^{-}\right)$, and $u_{\psi(x)}$ is the chart in $\mathcal{U}$ for the singularity $\psi(x)$; 
(2) if $u_{A}:\left(U_{A}, A\right) \rightarrow(\mathbb{C}-\operatorname{int}(D), \partial D)$ is the chart for a boundary component $A$, then there is a commutative diagra

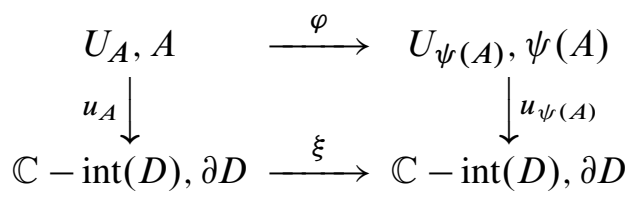

where $\xi=r_{(p, k, \lambda)}^{+} \circ \eta_{(p, k, \lambda)}^{\prime}$ or $\xi=r_{(p, k, \lambda)}^{-} \circ \eta_{(p, k, \lambda)}^{\prime}$ for some nonnegative integer $k$ (the boundary component $A$ is said to be of type $(p, k)^{+}$or of type $\left.(p, k)^{-}\right)$, and $u_{\psi(A)}$ is the chart in $\mathcal{U}$ for the boundary component $\psi(A)$.

The superscript + or - of the type indicates orientation preserving or reversing.

The local behavior and indices of isolated fixed point sets of a pseudo-Anosov homeomorphism are given as follow.

Lemma 3.2 [3, Lemma 2.1] Let $\psi$ be an orientation-preserving pseudo-Anosov homeomorphism on a compact surface $F$ with $\chi(F)<0$. Then there is a smooth atlas $\mathcal{U}$ of $F$ satisfying the conclusion of Lemma 3.1, and each fixed point of $\psi$ is included in one of the following cases:

(1) Isolated fixed point $x$.

(1.1) $x$ is of type $(p, 0)^{+}$with $\operatorname{ind}(\psi, x)=1-p$, where $p \geq 2$;

(1.2) $x$ is of type $(p, k)^{+}$with $p \nmid k$ with $\operatorname{ind}(\psi, x)=1$.

(2) Boundary component $C$ such that $\psi(C)=C$.

(2.1) $C$ is of type $(p, 0)^{+}$, and $C \subset \operatorname{Fix}(\psi)$ with $\operatorname{ind}(\psi, C)=-p$;

(2.2) $C$ is of type $(p, k)^{+}$with $p \nmid k$, and $C \cap \operatorname{Fix}(\varphi)=\varnothing$, hence ind $(\psi, C)=0$.

A fixed point in the interior which is not a singularity can be regarded as a " 2 -prong singularity", and hence is also included in this lemma. But the chart on it is not in the chosen atlas $\mathcal{U}$.

Lemma 3.3 [3, Lemma 3.1] Any orientation-preserving homeomorphism on an annulus $F_{0,2} \cong S^{1} \times I$ is isotopic to one of the following:

(1) an annular twist $\psi(z, t)=\left(z e^{2(a+b t) \pi i}, t\right)$, where $a$ and $b$ are rational numbers;

(2) a flip-twist $\psi(z, t)=\left(\bar{z} e^{a(1-2 t) \pi i}, 1-t\right)$, where $a$ is a rational number. 
Lemma 3.4 [3, Lemma 3.6] Let $M$ be a connected compact oriented surface $M$ with $\chi(M)<0$. Then any orientation-preserving homeomorphism on $M$ is isotopic to a homeomorphism $\psi$ (in standard form), having following properties. There is a set (the cutting system) $\Gamma=\left\{\gamma_{1}, \gamma_{2}, \ldots, \gamma_{k}\right\}$ of simple closed curves on $M$. Each $\gamma_{j}$ has a neighborhood $N\left(\gamma_{j}\right)$ homeomorphic to $S^{1} \times I$ such that

(i) the restriction $\left.\psi\right|_{N\left(\gamma_{j}\right)}$ of $\psi$ on each $N\left(\gamma_{j}\right)$ is an annular twist or a flip-twist;

(ii) the restriction of $\psi$ on each component of $M-\bigcup_{j=1}^{k}$ int $N\left(\gamma_{j}\right)$ is either periodic or pseudo-Anosov. (These components are said to be pieces, or $\psi$-pieces if we need to emphasis the related homeomorphism $\psi$.)

Moreover, each nonempty fixed point class of $\psi$ is a connected subset of $M$, being included in one of the following cases:

(1) Isolated fixed point $x$.

(1.1) $\psi$ is conjugate to a rotation in a neighborhood of $x$ in periodic piece, $\operatorname{ind}(\psi, x)=1$;

(1.1) a fixed point of an annular flip-twist, $\operatorname{ind}(\psi, x)=1$;

(1.1) a fixed point of type $(p, 0)^{+}$in a pseudo-Anosov piece, $\operatorname{ind}(\psi, x)=1-p$;

(1.1) a fixed point of $(p, k)^{+}$with $p \nmid k$ in a pseudo-Anosov piece, $\operatorname{ind}(\psi, x)=1$.

(2) Fixed point circle $C$.

(2.2) an isolated fixed point set of an annular twist; ind $(\psi, C)=0$;

(2.2) a boundary component with type $(p, 0)^{+}$of some pseudo-Anosov piece, on the other side $C$ is a boundary component of an annular twist; $\operatorname{ind}(\psi, C)=$ $-p$;

(2.2) a boundary component of $M$, and also a boundary component with type $(p, 0)^{+}$of some pseudo-Anosov piece, $\operatorname{ind}(\psi, C)=-p$;

(3) Fixed point subsurface.

Corollary 3.5 If $\psi$ is in standard form, then $\psi^{n}$ is in standard form for any positive $n$. Moreover, the cutting system of $\psi^{n}$ can be chosen as a subset of a cutting system of $\psi$.

Proposition 3.6 Let $\psi: M \rightarrow M$ be an orientation-preserving homeomorphism on a connect compact oriented surface $M$ with $\chi(M)<0$, and be in standard form. Let $V$ be an invariant set of $\psi^{n}$ which consists of some $\psi$-pieces and some neighborhoods of cutting curves. If $N\left(\left(\left.\psi\right|_{V}\right)^{n}\right)>0$, then $N\left(\psi^{n}\right)>0$. 


\section{Periodic homeomorphisms}

In this section, we shall discuss the upper bound for the free degree of periodic homeomorphisms on a compact surface.

Given a compact surface $F$, we write $o(F)$ for the maximal order of periodic homeomorphisms.

Lemma 4.1 (1) $o\left(F_{g, b}\right) \leq o\left(F_{g}\right)$ for all $b$.

(2) $o\left(N_{g, b}\right) \leq o\left(N_{g}\right)$ for all $b$.

Proof Regard the closed surface $F_{g}$ as the quotient space of $F_{g, b}$ by collapsing each boundary component to one point. We write $q: F_{g, b} \rightarrow F_{g}$ for this natural quotient map. Let $\psi$ be a periodic homeomorphism on $F_{g, b}$. Then it induces a periodic homeomorphism $\bar{\psi}$ on $F_{g}$, ie there is a commutative diagram

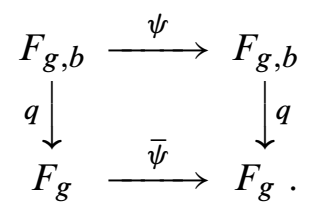

By definition, $o\left(F_{g}\right)$ is the maximum of the orders of the periodic map on $F_{g}$. The order of $\bar{\psi}$ is not greater than $o\left(F_{g}\right)$, ie there is a positive integer $n$ with $n \leq o\left(F_{g}\right)$ such that $\bar{\psi}^{n}$ is the identity on $F_{g}$. It follows that $\psi^{n}$ is the identity on $F_{g, b}$. This proves the conclusion (1). The proof of (2) is the same.

Lemma 4.2 Let $\xi$ be a self homeomorphism on a connected compact surface $M$ which is homotopic to a periodic map. If $\chi(M) \neq 0$, then there is a positive integer $n \leq o(M)$ such that $N\left(\xi^{n}\right)=1$, and hence the free degree $\mathfrak{f r}(\xi)$ of $\xi$ is no more than $o(M)$.

Proof This lemma is trivial if $o(M)$ is infinite.

Assume that $o(M)$ is finite. Let $\phi$ be a periodic map homotopic to the given map $\xi$. By definition of maximal order, there is natural number $n$ with $n \leq o(M)$ such that $\phi^{n}=$ id. From the homotopy invariance of Nielsen number, we have that $N\left(\xi^{n}\right)=$ $N\left(\phi^{n}\right)=N($ id $)$. Since $\chi(M) \neq 0$, we have that $N\left(\xi^{n}\right)=N\left(\phi^{n}\right)=N($ id $)=1$.

Combining our two lemmas with Wang [6, Theorem 1], we have:

Theorem 4.3 (1) Let $\xi: F_{g, b} \rightarrow F_{g, b}$ be a self-map homotopic to a periodic one. Then $\mathfrak{f r}(\xi) \leq 4 g+3+(-1)^{g}$ for all $g \geq 2$.

(2) Let $\xi: N_{g, b} \rightarrow N_{g, b}$ be a self-map homotopic to a periodic one. Then $\mathfrak{f r}(\xi) \leq$ $2 g-1+(-1)^{g+1}$ for all $g \geq 3$. 
Let us consider the free degree of compact surface with genus 0 or 1 . It is known that $\mathfrak{f r}\left(F_{0,0}\right)=2$, and $\mathfrak{f r}\left(N_{1,0}\right)=1$.

As for the cases $F_{0, b}, F_{1, b}, N_{1, b}$ and $N_{2, b}$, we have the following examples.

Example 4.4 Regard $F_{0,2}$ as

$$
S^{1} \times I=\{(z, t)|| z \mid=1,0 \leq t \leq 1\} .
$$

Given any positive integer $k$, a periodic homeomorphism $\xi_{k}$ is defined by $\xi_{k}(z, t)=$ $\left(z e^{2 \pi i / k}, t\right)$.

Pick a small open disk $W$ in $F_{0,2}$. Note that $G=F_{0,2}-\bigsqcup_{j=1}^{\infty} \xi_{k}^{j}(W)$ is homeomorphic to $F_{0, k+2}$. The restriction $\left.\xi_{k}\right|_{G}$ of $\xi_{k}$ on $G$ is also a periodic homeomorphism. Each point is a periodic point of period $k$. Hence, $\mathfrak{f r}\left(\left.\xi_{k}\right|_{G}\right)=k$. This implies that $\mathfrak{f r}\left(F_{0, k+2}\right) \geq k$.

Let $\tau: F_{0,2} \rightarrow F_{0,2}$ be an involution given by $\tau(z, t)=(-z, 1-t)$. It gives a $\mathbb{Z}_{2}$ action on $F_{0,2}$, and the orbit space $F_{0,2} / \tau$ is homeomorphic to the Möbius band $N_{1,1}$. If $k$ is even, $\xi_{k}$ induces a periodic homeomorphism $\bar{\xi}_{k}: F_{0,2} / \tau \rightarrow F_{0,2} / \tau$ with period $k / 2$. Note that each point on $\bar{\xi}_{k}$ has period $k / 2$. We have that $\mathfrak{f r}\left(\left.\bar{\xi}_{k}\right|_{G / \tau}\right)=k / 2$. Since $G / \tau \cong N_{1, k / 2+1}$, we also have that $\mathfrak{f r}\left(N_{1, k / 2+1}\right) \geq k / 2$.

Example 4.5 Regard $F_{1,0}$ as

$$
S^{1} \times S^{1}=\{(z, w)|| z|=| w \mid=1\} \subset \mathbb{C}^{2} .
$$

Given any positive integer $k$, a periodic homeomorphism $\xi_{k}$ is defined by $\xi_{k}(z, w)=$ $\left(z e^{2 \pi i / k}, w\right)$.

Pick a small open disk $W$ in $F_{1,0}$. Note that

$$
G=F_{1,0}-\bigsqcup_{j=1}^{\infty} \xi_{k}^{j}(W)=F_{1,0}-\bigsqcup_{j=1}^{k} \xi_{k}^{j}(W)
$$

is homeomorphic to $F_{1, k}$. The restriction $\left.\xi_{k}\right|_{G}$ of $\xi_{k}$ on $G$ is also a periodic homeomorphism. Each point is a periodic point of period $k$. Hence, $\mathfrak{f r}\left(\left.\xi_{k}\right|_{G}\right)=k$. This implies that $\mathfrak{f r}\left(F_{1, k}\right) \geq k$.

Let $\tau: F_{1,0} \rightarrow F_{1,0}$ be an involution given by $\tau(z, w)=(-z, \bar{w})$. It gives a $\mathbb{Z}_{2}$ action on $F_{1,0}$, and the orbit space $F_{1,0} / \tau$ is homeomorphic to the Klein bottle $N_{2,0}$. If $k$ is even, $\xi_{k}$ induces a periodic homeomorphism $\bar{\xi}_{k}: F_{1,0} / \tau \rightarrow F_{1,0} / \tau$ with period $k / 2$. Note that each point on $\bar{\xi}_{k}$ has period $k / 2$. We have that $\mathfrak{f} \mathfrak{r}\left(\left.\bar{\xi}_{k}\right|_{G / \tau}\right)=k / 2$. Since $G / \tau \cong N_{2, k / 2}$, we also have that $\mathfrak{f r}\left(N_{2, k / 2}\right) \geq k / 2$. 
Moreover, by using irrational angle rotation, we can show that there is a homeomorphism on $F_{0,2}$ (the annulus) without any periodic point, and a homeomorphism on $N_{1,1}$ (the Möbius band) without any periodic point. This implies that both $\mathfrak{f r}\left(F_{0,2}\right)$ and $\mathfrak{f r}\left(N_{1,1}\right)$ are infinite. But, by classical fixed point theorem, any map on $F_{0,1}$ (the disk) must have a fixed point. Hence, $\mathfrak{f} \mathfrak{r}\left(F_{0,1}\right)=1$.

\section{Special homeomorphisms on surfaces with small genus}

In this section, we consider the free degree of some homeomorphisms on the surfaces of genus 0 or 1 .

Lemma 5.1 Let $\xi: F_{0, b} \rightarrow F_{0, b}$ be an orientation preserving homeomorphism on a sphere with $b$ boundary components. If there are at least three boundary components on $F_{0, b}$ which are invariant under $\xi$, then $L(\xi) \neq 0$, hence $\mathfrak{f r}(\xi)=1$.

Proof See Figure 1.

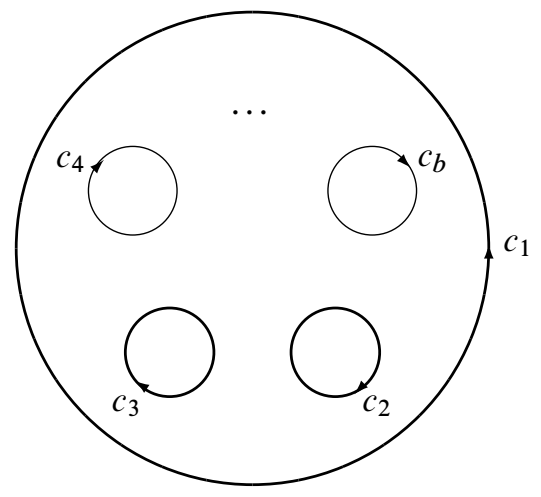

Figure 1: Bases of $H_{1}\left(F_{0, b}, \mathbb{Q}\right)$

The boundary components $c_{1}, c_{2}, c_{3}$ are invariant under $\xi$. Choose the classes $\left[c_{2}\right],\left[c_{3}\right]$, $\left[c_{4}\right], \ldots,\left[c_{b}\right]$ as a basis of $H_{1}\left(F_{0, b}, \mathbb{Q}\right)$. The induced isomorphism $\xi_{* 1}$ on $H_{1}\left(F_{0, b}, \mathbb{Q}\right)$ by $\xi$ has the form

$$
\left(\begin{array}{ccccc}
1 & 0 & 0 & 0 & 0 \\
0 & 1 & 0 & 0 & 0 \\
0 & 0 & B_{1} & 0 & 0 \\
0 & 0 & 0 & \ddots & 0 \\
0 & 0 & 0 & 0 & B_{l}
\end{array}\right),
$$

where $B_{1}, \ldots, B_{l}$ are permutation matrices.

So $\operatorname{tr}\left(\xi_{* 1}\right) \geq 2$ and $L(\xi)=1-\operatorname{tr}\left(\xi_{* 1}\right) \neq 0$. 
Lemma 5.2 Let $\xi: F_{1, b} \rightarrow F_{1, b}$ be an orientation preserving homeomorphism on a torus with $b \geq 1$ boundary components. If there is at least one boundary component of $F_{1, b}$ which is invariant under $\xi$, then $\mathfrak{f} \mathfrak{r}(\xi) \leq 6$.

Proof See Figure 2. The boundary component $c_{1}$ is invariant under $\xi$. Choose $[x],[y],\left[c_{2}\right], \ldots,\left[c_{b}\right]$ as bases of $H_{1}\left(F_{1, b}, \mathbb{Q}\right)$. The induced isomorphism $\xi_{* 1}$ on $H_{1}\left(F_{1, b}, \mathbb{Q}\right)$ by $\xi$ has the form

$$
\left(\begin{array}{ccccc}
a_{11} & a_{12} & \cdots & \cdots & \cdots \\
a_{21} & a_{22} & \cdots & \cdots & \cdots \\
0 & 0 & B_{1} & 0 & 0 \\
0 & 0 & 0 & \ddots & 0 \\
0 & 0 & 0 & 0 & B_{l}
\end{array}\right)
$$

where $B_{1}, \ldots, B_{l}$ are permutation matrices. This matrix is similar to

$$
\left(\begin{array}{ccccc}
\lambda & * & \cdots & \cdots & \cdots \\
0 & \lambda^{-1} & \cdots & \cdots & \cdots \\
0 & 0 & C_{1} & 0 & 0 \\
0 & 0 & 0 & \ddots & 0 \\
0 & 0 & 0 & 0 & C_{l}
\end{array}\right)
$$

Here if $C_{j}$ is of rank $n$, then

$$
C_{j}=\left(\begin{array}{cccc}
e^{2 \pi / n i} & 0 & 0 & 0 \\
0 & \ddots & 0 & 0 \\
0 & 0 & e^{2(n-1) \pi / n i} & \\
0 & 0 & 0 & 1
\end{array}\right)
$$

Suppose the number of rank 1,2,3,4,6 $C_{j}$ 's is $m, t, s, q, r$. If $L\left(\xi^{1}\right)=L\left(\xi^{2}\right)=L\left(\xi^{3}\right)=$ $L\left(\xi^{4}\right)=L\left(\xi^{6}\right)=0$, then we have the following identities.

$$
\begin{aligned}
\lambda+\lambda^{-1}+m & =1 \\
\lambda^{2}+\lambda^{-2}+m+2 t & =1 \\
\lambda^{3}+\lambda^{-3}+m+3 s & =1 \\
\lambda^{4}+\lambda^{-4}+m+2 t+4 q & =1 \\
\lambda^{6}+\lambda^{-6}+m+2 t+3 s+6 r & =1
\end{aligned}
$$

So $(1-m)^{2}=\left(\lambda+\lambda^{-1}\right)^{2}=\lambda^{2}+\lambda^{-2}+2=3-m-2 t$. We have $m=2, t=0$ or $m=1, t=1$ or $m=0, t=1$. 
If $m=2, t=0$, then $\lambda+\lambda^{-1}+1=0$ induces $\lambda=e^{\frac{2 \pi}{3} i}$ or $e^{-\frac{2 \pi}{3} i}$. We have $\lambda^{3}+\lambda^{-3}=2$ which contradicts (5-3).

If $m=1, t=1$, then $\lambda+\lambda^{-1}=0$ induces $\lambda=i$ or $-i$. We have $\lambda^{4}+\lambda^{-4}=2$ which contradicts (5-4).

If $m=0, t=1$, then $\lambda+\lambda^{-1}-1=0$ induces $\lambda=e^{\pi / 3 i}$ or $e^{-\pi / 3 i}$. We have $\lambda^{6}+\lambda^{-6}=2$ which contradicts (5-5).

The argument above shows that at least one of the $L\left(\xi^{1}\right), L\left(\xi^{2}\right), L\left(\xi^{3}\right), L\left(\xi^{4}\right)$, $L\left(\xi^{6}\right)$ is not equal to 0 . Thus $\mathfrak{f r}(\xi) \leq 6$.

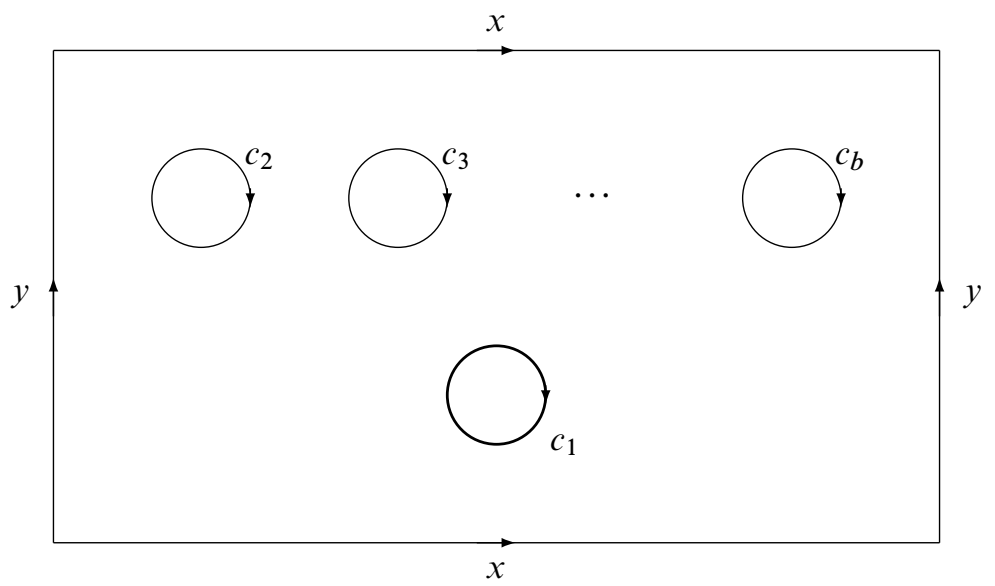

Figure 2: Bases of $H_{1}\left(F_{1, b}, \mathbb{Q}\right)$

\section{Pseudo-Anosov homeomorphisms}

We consider the free degrees of pseudo-Anosov homeomorphisms in this section.

Lemma 6.1 Let $\mathcal{F}$ be a singular foliation on a compact surface $F_{g, b}$. Then

$$
\sum_{m=1}^{\infty}\left(\left(1-\frac{m}{2}\right) \operatorname{Pr}_{m}^{\mathrm{int}}(\mathcal{F})-\frac{m}{2} \operatorname{Pr}_{m}^{\mathrm{bd}}(\mathcal{F})\right)=\chi\left(F_{g, b}\right)=2-2 g-b,
$$

where $\operatorname{Pr}_{m}^{\mathrm{int}}(\mathcal{F})$ is the number of $m$-prong singularities in the interior of $F_{g, b}$ and $\operatorname{Pr}_{m}^{\mathrm{bd}}(\mathcal{F})$ is the number of boundary components of $F_{g, b}$ with $m$-prong singularities. 
Proof Consider the closed surface $F_{g}$, a singular foliation can be regarded as a path field on $F_{g}$. Both have the same singularity set. An $m$-prong singularity has index $1-m / 2$.

Lemma 6.2 Let $\xi: F_{g, b} \rightarrow F_{g, b}$ be a self homeomorphism on $F_{g, b}(g \geq 2)$ which is homotopic to an orientation preserving pseudo-Anosov homeomorphism $\psi$. If the stable (and hence unstable) singular foliation of $\psi$ has no 1-prong singularity, then $\mathfrak{f} \mathfrak{r}(\xi) \leq 8 g-8$.

Proof Since Nielsen number is a homotopy invariant, we only need to prove that there exists a positive integer $n \leq 8 g-8$ such that $N\left(\psi^{n}\right)>0$.

Let $\mathcal{F}^{s}$ and $\mathcal{F}^{u}$ be respectively stable and unstable singular foliations of the pseudoAnosov homeomorphism $\psi$. We denote the number of $m$-prong singularities of $\mathcal{F}^{s}$ by $\operatorname{Pr}_{m}\left(\mathcal{F}^{s}\right)$. Regard the closed surface $F_{g}$ as the quotient space of $F_{g, b}$ by collapsing each boundary component to one point. Then $\psi$ induces an orientation preserving homeomorphism $\bar{\psi}$ on $F_{g}$, satisfying the commutative diagram

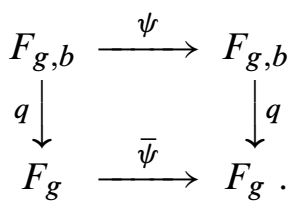

Since $\mathcal{F}^{s}$ has no 1-prong singularity, the quotient map $q$ gives a singular foliation $q\left(\mathcal{F}^{s}\right)$ on $F_{g}$. Thus, $q\left(\mathcal{F}^{s}\right)$ and $q\left(\mathcal{F}^{u}\right)$ are respectively stable and unstable singular foliations of the pseudo-Anosov homeomorphism $\bar{\psi}$.

By the results of Nielsen [4] with Dicks and Llibre [1] (see Wang [7, Remark (1)]), we know that $\mathfrak{f r}(\bar{\psi})=2 g-2$. This implies that there must be an integer $n_{0}$ with $n_{0} \leq 2 g-2$ such that $\bar{\psi}^{n_{0}}$ has a fixed point $\bar{x}_{0}$. Since $\bar{\psi}^{n_{0}}$ is a pseudo-Anosov homeomorphism on the closed surface $F_{g}$, the point $\bar{x}_{0}$ consists of a fixed point class of $\bar{\psi}^{n_{0}}$ with $\operatorname{ind}\left(\bar{\psi}^{n_{0}}, \bar{x}_{0}\right) \neq 0$. If $q^{-1}\left(\bar{x}_{0}\right)$ is a singleton, the point $q^{-1}\left(\bar{x}_{0}\right)$ is an isolated fixed point of $\psi^{n_{0}}$ with nonzero index. Since $\psi$ is in standard form, this isolated fixed point is an essential fixed point class of $\psi^{n_{0}}$. Thus, we have that $N\left(\psi^{n_{0}}\right)>0$.

If it is not a singleton, $q^{-1}\left(\bar{x}_{0}\right)$ is a boundary component of $F_{g, b}$. Thus, $q^{-1}\left(\bar{x}_{0}\right)$ is an invariant circle of type $\left(p_{0}, k_{0}\right)$ for $\psi^{n_{0}}$. Note that $q^{-1}\left(\bar{x}_{0}\right)$ is a fixed point circle for $\psi^{p_{0} n_{0}}$ of type $\left(p_{0}, p_{0} k_{0}\right)$, ie of type $\left(p_{0}, 0\right)$. By Lemma 3.2, we have that $\operatorname{ind}\left(\psi^{p_{0} n_{0}}, q^{-1}\left(\bar{x}_{0}\right)\right)=-p_{0} \neq 0$. It follows that $N\left(\psi^{p_{0} n_{0}}\right)>0$. It is sufficient to show that $p_{0} n_{0} \leq 8 g-8$. There are two cases: 
Case $1 p_{0}=2$ or 3 . We have that $p_{0} n_{0} \leq 3(2 g-2)=6 g-6$.

Case $2 p_{0} \geq 4$. Applying Lemma 6.1 to the foliation $q\left(\mathcal{F}^{s}\right)$ on $F_{g}$, we have

$$
2-2 g=\chi\left(F_{g}\right)=\sum_{p=1}^{\infty}\left(1-\frac{p}{2}\right) \operatorname{Pr}_{p}^{\mathrm{int}}\left(q\left(\mathcal{F}^{s}\right)\right) \leq\left(1-\frac{p_{0}}{2}\right) \operatorname{Pr}_{p_{0}}^{\mathrm{int}}\left(q\left(\mathcal{F}^{s}\right)\right),
$$

because $q\left(\mathcal{F}^{S}\right)$ has no 1 -prong singularity. It follows that

$$
p_{0} \operatorname{Pr}_{p_{0}}^{\text {int }}\left(q\left(\mathcal{F}^{s}\right)\right) \leq(4 g-4)\left(1+\frac{2}{p_{0}-2}\right) \leq 8 g-8 .
$$

Since $\psi$ permutes the boundaries of $p_{0}$-prong, we have that

$$
n_{0} \leq \operatorname{Pr}_{p_{0}}^{\mathrm{bd}}\left(\mathcal{F}^{s}\right) \leq \operatorname{Pr}_{p_{0}}^{\mathrm{bd}}\left(\mathcal{F}^{s}\right)+\operatorname{Pr}_{p_{0}}^{\mathrm{int}}\left(\mathcal{F}^{s}\right)=\operatorname{Pr}_{p_{0}}^{\mathrm{int}}\left(q\left(\mathcal{F}^{s}\right)\right)
$$

and hence $p_{0} n_{0} \leq 8 g-8$.

\section{Main results}

Lemma 7.1 Let $F_{g, b}$ be a connected compact surface of genus $g$ with $b$ boundary components, where $g \geq 2$. Then for any orientation-preserving homeomorphism $\psi: F_{g, b} \rightarrow F_{g, b}$, there is a positive integer $n$ with $n \leq 12 g-12$ such that $N\left(\psi^{n}\right)>0$.

Proof The procedure of our proof will be fulfilled by using a reduction on the pairs $(g, b)$ according to the lexicographic order. That is, we say $\left(g^{\prime}, b^{\prime}\right)<\left(g^{\prime \prime}, b^{\prime \prime}\right)$ if either $g^{\prime}<g^{\prime \prime}$ or $g^{\prime}=g^{\prime \prime}$ and $b^{\prime}<b^{\prime \prime}$.

By the homotopy invariance of Nielsen number, we may assume that $\psi$ is in standard form.

Case $1 b=0$. From the proof of Wang [7, Theorem 1], we know that the Lefschetz number $L\left(\psi^{n}\right)$ is nonzero for some $n$ satisfying $n \leq 4$ if $g=2 ; n \leq 2 g-2$ if $g \geq 3$. This implies that $N\left(\psi^{n}\right)>0$ for such an $n$.

Case $2 \psi$ is periodic. We have $N\left(\psi^{n}\right)=1$ for some $n \leq 4 g+3+(-1)^{g}$.

Case $3 \psi$ is a pseudo-Anosov map. Note that the homeomorphism $\psi$ permutes the boundary components of type $(1,0)^{+}$. Let $l_{0}$ be the minimal length of orbits of $\psi$-action on the set of all boundary components of type $(1,0)^{+}$. We have three subcases according to the value of $l_{0}$.

Subcase 3.1 $l_{0}=0$. This means logically that the number of boundary components of $F_{g, b}$ with type $(1,0)$ is zero. This is done in Lemma 6.2. 
Subcase 3.2 $0<l_{0} \leq 12 g-12$. Let $C_{0}$ be a boundary component with type $(1,0)^{+}$ such that $\psi^{l_{0}}\left(C_{0}\right)=C_{0}$. By Lemma 3.4, $C_{0}$ is a fixed point class of $\psi^{l_{0}}$, having fixed point index -1 . This implies that $N\left(\psi^{l_{0}}\right)>0$.

Subcase 3.3 $l_{0}>12 g-12$. We collapse each boundary component of type $(1,0)^{+}$ to one point. The homeomorphism $\psi$ induces a homeomorphism $\bar{\psi}$ on the resulting surface $F_{g, b^{\prime}}$. We write $q: F_{g, b} \rightarrow F_{g, b^{\prime}}$ for this natural quotient map. Then we have a commutative diagram

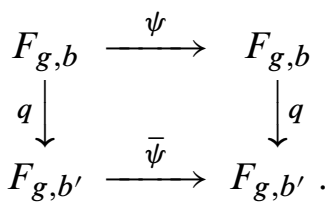

Of course, $\bar{\psi}$ is not in standard form. By definition of $l_{0}$, we have that $\operatorname{Fix}\left(\psi^{m}\right)=$ Fix $\left(\bar{\psi}^{m}\right)$ for any $m$ with $0<m \leq 12 g-12$. Since $q$ is a homeomorphism near this fixed point set, any isolated fixed point set have the same fixed point indices. In other word, $\operatorname{ind}\left(\psi^{m}, F\right)=\operatorname{ind}\left(\bar{\psi}^{m}, F\right)$ for any fixed point class $F$ of $\psi^{m}$ with $0<m \leq 12 g-12$. Since $q$ is a surjective map and since $q$ only collapses $(1,0)^{+}$ boundary components, any fixed point class of $\bar{\psi}^{m}$ will be an union of some fixed point classes of $\psi^{m}$. Any essential fixed point class of $\bar{\psi}^{m}$ contains at least one essential fixed point class of $\psi^{m}$ if $m \leq 12 g-12$. Thus, it is sufficient to prove that $N\left(\bar{\psi}^{m}\right)>0$ for some $m$ with $m \leq 12 g-12$. This is just the inductive assumption.

Case $4 \psi$ is reducible. Let $P_{0}$ be a reduced piece with the biggest genus among all pieces. Assume that $P_{0} \cong F_{g_{0}, b_{0}}$.

Thus, either $g_{0}<g$ or $g_{0}=g$ and $b_{0}<b$. Note that $\psi$ permutes all pieces.

We consider three subcases according to the value of $g_{0}$.

Case 4.1 $g_{0} \geq 2$. We write $l_{0}$ for the orbit length of $P_{0}$ under the action of $\psi$. That is $\psi^{l_{0}}\left(P_{0}\right)=P_{0}$, and $\psi^{j}\left(P_{0}\right) \neq P_{0}$ for $j=1,2, \ldots, l_{0}-1$. Clearly, $\left(\left.\psi\right|_{P_{0}}\right)^{l_{0}}$ is a homeomorphism on $P_{0} \cong F_{g_{0}, b_{0}}$. By assumption of reduction, there is a positive number $n_{0}$ with $n_{0} \leq 12 g_{0}-12$ such that $\left.\left.N\left(\left(\psi||_{P_{0}}\right)^{l_{0}}\right)^{n_{0}}\right)\right)>0$, ie $N\left(\left.\psi^{l_{0} n_{0}}\right|_{P_{0}}\right)>0$. By Proposition 3.6, we have that $N\left(\psi^{l_{0} n_{0}}\right)>0$. Clearly,

$$
l_{0} n_{0} \leq l_{0}\left(12 g_{0}-12\right) \leq 12 g-12 l_{0} \leq 12 g-12 .
$$

Case 4.2 $g_{0}=0$ or 1 . Consider the quotient map $q: F_{g, b} \rightarrow F_{g}$ and the induced homeomorphism satisfying the commutative diagram (4-1). Let $\Gamma=\left\{\gamma_{1}, \gamma_{2}, \ldots, \gamma_{k}\right\}$ be the cutting system for $\psi$. We assume that $q\left(\gamma_{j}\right)$ is essential in $F_{g}$ for $j=1,2, \ldots k^{\prime}$, and inessential for $j=k^{\prime}+1, \ldots, k$. We write $\Gamma^{\prime}=\left\{\gamma_{1}, \gamma_{2}, \ldots, \gamma_{k^{\prime}}\right\}$. Then each component of $F_{g}-q\left(\Gamma^{\prime}\right)$ is an union of one component of $F_{g}-q(\Gamma)$ and other 
components of $F_{g}-q(\Gamma)$ are disks. This implies that $k^{\prime}>0$ and the maximal genus of the components of $F_{g}-q\left(\Gamma^{\prime}\right)$ is still $g_{0}$. Since each curve $q\left(\gamma_{j}\right)$ in $q\left(\Gamma^{\prime}\right)$ is essential in $F_{g}$, the Euler characteristic number of each component of $F_{g}-q\left(\Gamma^{\prime}\right)$ is negative. Consider two sub-subcases.

Case 4.2.1 $g_{0}=1$. Let $Q_{0}$ be a component of $F_{g}-q\left(\Gamma^{\prime}\right)$ with genus 1 . Let $l_{0}$ be the orbit length of $Q_{0}$ under the action of $\bar{\psi}$, and $b_{0}$ be number of boundary components of $Q_{0}$. Note that each component of $F_{g}-q\left(\Gamma^{\prime}\right)$ has nonpositive Euler characteristic number. We obtain that $l_{0}\left(2-2-b_{0}\right) \geq 2-2 g$. Since $\bar{\psi}^{l_{0}}\left(Q_{0}\right)=Q_{0}$, there must be a positive integer $n_{0}$ with $n_{0} \leq b_{0}$ such that $\bar{\psi}^{l_{0} n_{0}}$ has at least one invariant boundary component $C_{0}$ of $Q_{0}$. By commutative diagram (4-1), we have $\psi^{l_{0} n_{0}}\left(q^{-1}\left(Q_{0}\right)\right)=$ $q^{-1}\left(Q_{0}\right)$. By definition of $q$, the closure $P=\overline{q^{-1}\left(Q_{0}\right)}$ of $q^{-1}\left(Q_{0}\right)$ is homeomorphic to $F_{1, b}$ for some $b \geq b_{0}$. Apply Lemma 5.2 to the homeomorphism $\left.\psi^{l_{0} n_{0}}\right|_{P}$, there is a positive integer $n$ with $n \leq 6$ such that $L\left(\left(\left.\psi^{l_{0} n_{0}}\right|_{P}\right)^{n}\right) \neq 0$. Hence, $N\left(\left(\left.\psi^{l_{0} n_{0}}\right|_{P}\right)^{n}\right)>0$. It follows from Proposition 3.6 that $N\left(\psi^{l_{0} n_{0} n}\right)>0$.

Case 4.2.2 $g_{0}=0$. In this situation, each component of $F_{g}-q\left(\Gamma^{\prime}\right)$ has genus zero, ie a disk with holes. Note that each component of $F_{g}-q\left(\Gamma^{\prime}\right)$ has nonpositive Euler characteristic number. From the additivity of Euler characteristic numbers, there must be a component $Q_{0}$ with $\chi\left(Q_{0}\right)<0$, ie $Q_{0}$ has at least three boundary components. Let $l_{0}$ be the orbit length of $Q_{0}$ under the action of $\bar{\psi}$, and $b_{0}$ be number of boundary components of $Q_{0}$. Then we have $l_{0}\left(2-b_{0}\right) \geq 2-2 g$. This implies that

$$
l_{0} b_{0} \leq \frac{b_{0}}{b_{0}-2}(2 g-2) \leq 6 g-6,
$$

because $b_{0} \geq 3$. Since $\bar{\psi}^{l_{0}}$ permutes the boundary components of $Q_{0}$, there must be a positive integer $n_{0}$ with $n_{0} \leq b_{0}$ such that $\bar{\psi}^{l_{0} n_{0}}$ fixes set-wisely at least three boundary components. Notice that the closure $P=\overline{q^{-1}\left(Q_{0}\right)}$ of $q^{-1}\left(Q_{0}\right)$ is also a disk with holes. The homeomorphism $\left.\psi^{l_{0} n_{0}}\right|_{P}$ also fixes set-wisely at least three boundary components. Apply Lemma 5.1 to the homeomorphism $\left.\psi^{l_{0} n_{0}}\right|_{P}$, we have that $L\left(\left.\psi^{l_{0} n_{0}}\right|_{P}\right) \neq 0$. Hence, $N\left(\left.\psi^{l_{0} n_{0}}\right|_{P}\right)>0$. It follows from Proposition 3.6 that $N\left(\psi^{l_{0} n_{0}}\right)>0$.

Theorem 7.2 For $F_{g, b}$ and $N_{g, b}$ orientable and nonorientable genus $g$ surfaces with $b$ boundary components, the free degrees satisfy:

$$
\begin{gathered}
\max _{b} \mathfrak{f r}\left(F_{g, b}\right) \begin{cases}=\infty & \text { if } g=0,1, \\
\leq 24 g-24 & \text { if } g \geq 2 .\end{cases} \\
\max _{b} \mathfrak{f} \mathfrak{r}\left(N_{g, b}\right) \begin{cases}=\infty & \text { if } g=1,2, \\
\leq 12 g-24 & \text { if } g \geq 3 .\end{cases}
\end{gathered}
$$


Proof The infiniteness has been shown in Example 4.4 and Example 4.5.

Consider a homeomorphism $\psi: F_{g, b} \rightarrow F_{g, b}$, where $g \geq 2$. Then $\psi^{2}$ must be orientation preserving. By Lemma 7.1, there is a positive integer $n$ with $n \leq 12 g-12$ such that $N\left(\psi^{2 n}\right)=N\left(\left(\psi^{2}\right)^{n}\right)>0$. It follows that $\psi^{2 n}$ has a fixed point. Hence, $\mathfrak{f r}(\psi) \leq 24 g-24$. Since $\psi$ is an arbitrary homeomorphism on $F_{g, b}$. We obtain that $\mathfrak{f r}\left(F_{g, b}\right) \leq 24 g-24$.

Let $\eta: F_{g-1,2 b} \rightarrow N_{g, b}$ be the classical orientation covering. Write $\tau$ for the unique nontrivial covering transformation. Any homeomorphism $\psi: N_{g, b} \rightarrow N_{g, b}$ has two liftings $\phi$ and $\tau \phi$. Without loss of the generality, we may assume that $\phi$ is orientation preserving. By Lemma 7.1, there is a positive integer $n$ with $n \leq 12(g-1)-12$ such that $\phi^{n}$ has a fixed point $x_{0}$. Clearly, $\psi^{n}\left(\eta\left(x_{0}\right)\right)=\eta\left(\phi^{n}\left(x_{0}\right)\right)=\eta\left(x_{0}\right)$, ie $\eta\left(x_{0}\right)$ is a fixed point of $\psi^{n}$. This implies that $\mathfrak{f r}\left(N_{g, b}\right) \leq 12(g-1)-12=12 g-24$.

From the proof of this theorem, we obtain:

Corollary 7.3 For $F_{g, b}$ an orientable genus $g$ surface with $b$ boundary components, the orientation preserving free degree satisfies:

$$
\max _{b} \mathfrak{f r}^{+}\left(F_{g, b}\right) \begin{cases}=\infty & \text { if } g=0,1 \\ \leq 12 g-12 & \text { if } g \geq 2\end{cases}
$$

Acknowledgments The paper was enhanced by the referee's comments. The authors would like to thank professor Shicheng Wang for drawing them to this topic. The first author is supported by NSFC grant number 11001190. The second author is supported by NSFC grant number 10931005 .

\section{References}

[1] W Dicks, J Llibre, Orientation-preserving self-homeomorphisms of the surface of genus two have points of period at most two, Proc. Amer. Math. Soc. 124 (1996) 1583-1591 MR1301020

[2] B J Jiang, Lectures on Nielsen fixed point theory, Contemporary Math. 14, Amer. Math. Soc. (1983) MR685755

[3] B J Jiang, J H Guo, Fixed points of surface diffeomorphisms, Pacific J. Math. 160 (1993) 67-89 MR1227504

[4] J Nielsen, Fixed point free mappings, Mat. Tidsskr. B. 1942 (1942) 25-41 MR0013308 In German 
[5] W P Thurston, On the geometry and dynamics of diffeomorphisms of surfaces, Bull. Amer. Math. Soc. (N.S.) 19 (1988) 417-431 MR956596

[6] S C Wang, Maximum orders of periodic maps on closed surfaces, Topology Appl. 41 (1991) 255-262 MR1135102

[7] S C Wang, Free degrees of homeomorphisms and periodic maps on closed surfaces, Topology Appl. 46 (1992) 81-87 MR1177165

Department of Mathematics, Soochow University

Suzhou 215006, China

Department of Mathematics \& Institute of Mathematics and Interdisciplinary Science, Capital Normal University

Beijing 100048, China

wujianchun@suda.edu.cn, zhaoxve@mail.cnu.edu.cn

Received: 30 March $2011 \quad$ Revised: 6 August 2011 